\title{
Indicadores gráficos de la prueba del Dibujo de la Figura Humana en adolescentes hombres y mujeres de 12 a 16 años víctimas de agresiones sexuales
}

\author{
Graphic indicators in the test of Human Figure \\ Drawing (HFD) among adolescents from 12 to 16 years, \\ of both sexes, who have suffered sexual assault
}

Virginia Opazo Baeza', Javiera Rivera Toledo

Resumen

El presente estudio tiene por objetivo determinar la existencia de indicadores gráficos diferenciales en la prueba del Dibujo de la Figura Humana (DFH), entre adolescentes de 12 a 16 años, de ambos sexos, que hayan sufrido una agresión sexual crónica intrafamiliar o por parte de un conocido extrafamiliar, de aquellos en que no se sospeche dicha agresión. Para ello se evaluaron 130 indicadores gráficos, de los cuales 17 resultaron significativos considerando la variable "agresión sexual". De éstos, 3 se presentan con mayor frecuencia en el grupo agredido sexualmente, y los 14 indicadores restantes se presentan con mayor frecuencia en el grupo control. Además, se observaron respuestas diferenciales considerando la variable sexo en cada grupo de estudio.

Palabras clave: Agresión sexual, Prueba del Dibujo de la Figura Humana, Adolescencia.

\section{Abstract}

The present study aims to determine the existence of differential graphic indicators in the test of Human Figure Drawing (HFD) among

1 Virginia Opazo Baeza, Psicóloga, Universidad de Chile. Santiago, Chile.ps.virginia.opazo@gmail.com 2 Javiera Rivera Toledo, Psicóloga, Universidad de Chile. Santiago, Chile. ps.javierarivera@gmail.com 
adolescents from 12 to 16 years, of both sexes, who have suffered chronic sexual assault by a kin or by a known person outside the family, those that are not suspected of such aggression. To do that, 130 graphic indicators were evaluated, of which 17 were significant considering the variable "sexual assault". Of these, 3 were present most frequently in sexually abused group and the 14 other remaining indicators are presented most frequently in the control group. Furthermore, differential responses were observed considering the sex variable in each study group.

Key words: Sexual Assault, Test of Human Figure Drawing, Adolescence.

Agresión sexual infanto-juvenil en Chile

Dentro de todas las figuras que constituyen el maltrato infantil, la agresión sexual es considerada como una de las que genera consecuencias de mayor gravedad y que trastoca más áreas del desarrollo evolutivo de la persona. Es por esto que el Gobierno de Chile ha asumido el imperativo ético de terminar con este tipo de vulneración, incorporando en sus políticas públicas diferentes iniciativas que apuntan a la prevención, detección y reparación de las agresiones sexuales.

Cifras entregadas por instituciones como el Servicio Médico Legal, Carabineros de Chile y la Policía de Investigaciones de Chile, dan cuenta que se producen aproximadamente 30.000 atentados sexuales al año (Bain \& Yáńez, 2002), denunciándose sólo entre un 20\% y 25\% de los casos, disminuyendo las denuncias en el caso de nińas menores de 18 años (Ministerio de Salud, 1998). Estas instituciones además revelan que la mayoría de las víctimas corresponderían a menores de edad y que el $80 \%$ de las víctimas son de sexo femenino, teniendo un carácter repetitivo y crónico (Bain \& Yáñez, 2002). Sin embargo, cabe mencionar que los nińos y adolescentes de sexo masculino develan en menor medida la agresión sexual, por lo que se estima que, si todas las agresiones contra éstos se develaran, las cifras en relación al sexo podrían igualarse (Servicio Nacional de Menores, 2008). 
Otras investigaciones (Corsi, 1994) dan cuenta que más de un $80 \%$ de las agresiones sexuales infantiles son cometidas por una persona conocida por el niño o niña, siendo el $90 \%$ de estos casos un victimario de sexo masculino. Sin embargo, se debe tener en cuenta que no existen estudios unificados ni centralizados a nivel nacional con respecto a los delitos sexuales en la población infanto-juvenil, sino más bien existen datos parciales de distintas instituciones o diferentes estudios aislados que, como los señalados, definen de distintas maneras lo que corresponde a violación y/o abuso sexual, lo que impediría un análisis estadístico exhaustivo y global de la ocurrencia de estos fenómenos, y una real estimación de su magnitud.

Síntomas en adolescentes agredidos sexualmente

En relación a los indicadores que pondrían en evidencia las consecuencias que el hecho traumático produce tanto en el cuerpo como en el psiquismo, éstos pueden ser de naturaleza física (lesiones) como emocional. Ambos indicadores pueden estar estrechamente relacionados con la agresión sexual (indicadores específicos) o pueden acompañar a otros trastornos (indicadores inespecíficos). Generalmente la confirmación de la agresión sexual se basa en la presencia de más de uno de estos indicadores (Intebi, 1998).

Se debe considerar, además, que los indicadores emocionales como consecuencia de la agresión sexual pueden ser de dos tipos: por un lado, los que surgen como consecuencia de la agresión misma, y por otro, las implicancias emocionales de situaciones de victimización secundaria en los casos que ha habido una revelación mal manejada por la familia, amigos y/o instituciones (Llanos \& Sinclair, 2001; Policía de Investigaciones de Chile, 2003). A esta distinción es posible agregar un tercer tipo, los síntomas producidos por problemáticas preexistentes de la víctima, los cuales podrían ser gatillados por la agresión sexual.

En los adolescentes agredidos sexualmente se observa diversa sintomatología, la cual da cuenta del daño emocional que provoca el hecho traumático. En este sentido, algunos autores (Intebi, 1998; De Corral 
\& Echeburúa, 2006; Policía de Investigaciones de Chile, 2003; Barudy, 1998) diferencian entre síntomas funcionales, conductuales y sociales, emocionales y sexuales. Dentro de los síntomas funcionales se destacan los problemas de sueño y/o pesadillas, y cambios en los hábitos de comida. En relación a los síntomas conductuales y sociales se pueden mencionar un bajo rendimiento académico, conductas suicidas o autolesivas, las cuales tienen a la base una búsqueda desenfrenada de castigo, retraimiento social y conductas antisociales (fuga del hogar, vagancia, consumo de alcohol y drogas).

Con respecto a los síntomas emocionales destacan la hostilidad y la agresividad, sentimientos de culpa y vergüenza, depresión, ansiedad, baja autoestima y sentimientos de estigmatización, rechazo del propio cuerpo, desconfianza y rencor hacia los adultos, y síntomas relacionados con un trastorno por estrés postraumático.

Finalmente, dentro de los síntomas sexuales encontramos un conocimiento sexual precoz o inapropiado para su edad, masturbación compulsiva, excesiva curiosidad sexual y problemas de identidad sexual, comportamiento sexual precoz y embarazo adolescente.

Se observa una centralidad de síntomas en la esfera corporal, donde se evidencia una imagen corporal deteriorada por los efectos traumáticos de la experiencia abusiva, es decir, al producir la agresión sexual un daño en el cuerpo de la víctima (el cuerpo como objeto de vulneración), ésta elabora una imagen corporal deformada, sintiendo su cuerpo como algo malo, sucio, dañado, causal de vergüenza, tendiendo a rechazarlo (Girardi \& Pool, 2005; Zegers, 1981, en Carvacho, 1998).

Además de todos los síntomas ya mencionados, la agresión sexual generaría un gasto de energía dirigida al proceso de adaptación luego de la ruptura generada por el hecho abusivo (Barudy, 1998). Esto llevaría a que el o la adolescente se aleje de los procesos de desarrollo propios de su etapa evolutiva, centrando sus energías en la elaboración de vivencias y experiencias asociadas a la agresión sexual.

Cabe mencionar que la agresión sexual presenta una especial gravedad en la adolescencia, debido a la alta probabilidad de que el agresor realice contacto genital y penetración sexual, y con ello exista un riesgo real 
de embarazo. Se suma a esto la posibilidad de que el o la adolescente tome conciencia del alcance de la vulneración vivida (De Corral \& Echeburúa, 2006; Policía de Investigaciones de Chile, 2003).

\section{Adolescencia y género en agresión sexual}

Por la particularidad de la adolescencia se desprende que la elaboración de una agresión sexual tiene características diferentes a la efectuada en otros grupos etários. Además, algunos autores dan cuenta de sintomatología distinta según el sexo de la víctima. En este sentido, Intebi (1998) señala que los hombres tienden a presentar un patrón externalizante (conductas agresivas, disruptivas, etc.), mientras que las mujeres presentarían un patrón internalizante, donde la agresión es puesta en sí misma, por lo que se manifiestan más trastornos depresivos y de inhibición. Sin embargo, en ambos casos, se encuentra a la base un problema de autoestima y de depresión.

En relación a esto, otras investigaciones (Servicio Nacional de Menores, 2008) han dado cuenta que para los niños y adolescentes varones abusados es más difícil configurarse como una víctima que para las mujeres, ya que están más expuestos a identificarse con el modelo más agresivo de expresión de la violencia, mientras que las niñas son más proclives a aprender el modelo de indefensión. Por lo tanto, se podría deducir que la variable género influiría en los síntomas asociados a una agresión sexual.

Por otra parte, la variable género influiría también en la magnitud de los síntomas, esto se debería a que los adolescentes hombres agredidos sexualmente, al oponer mayor resistencia física que las mujeres frente a una agresión sexual, estarían más expuestos a distintos tipos de maltrato físico, lo que potenciaría aún más los efectos del acto traumático. Lo mencionado anteriormente se comprende desde una perspectiva de género, entendiendo lo femenino y lo masculino como categorías construidas cultural y contextualmente. De esta manera, se explicarían las diferencias en el comportamiento de hombres y mujeres, desarrolladas mediante un 
aprendizaje social. Por lo tanto, el impacto que tiene la agresión en los nińos, niñas y adolescentes está mediatizado por una serie de mensajes propios de la socialización de género.

De acuerdo a lo anterior, se desprende la relevancia del género como variable a considerar al momento de abordar la temática de la agresión sexual infanto-juvenil. Sin embargo existen pocos estudios que la contemplan.

Evaluación psicológica de las agresiones sexuales

A pesar de que la agresión sexual se relaciona con síntomas específicos como los ya mencionados, no existe un síndrome de agresión sexual infantil unívoco y formalmente reconocido. Esto surge como una problemática en el proceso de validación de un diagnóstico de agresión sexual (Intebi, 1998), junto con otras dificultades tales como: que la naturaleza de este delito lo convierte en un evento privado, raramente hay testigos más allá del agresor y la víctima, y que en la mayoría de los casos no existen huellas físicas de la agresión sexual, lo cual dificultaría la comprobación médica del ilícito (Chia, Fernández, Navarro, Salgado \& Valdés, 2005; Intebi, 1998). Debido a esto, el sistema judicial debe recurrir a otros elementos, datos y herramientas para confirmar la veracidad de la denuncia, considerando como prueba fundamental la declaración de la víctima (Alhambra, Huerta, Miranda, Navarro \& Prado, 2004).

La evaluación pericial de una Agresión Sexual Infantil se construye sobre tres pilares fundamentales que recogen información acerca de los siguientes tópicos: contexto, análisis de la credibilidad del niño y psicodiagnóstico (Maffioletti \& Salinas, 2005). Es dentro de este último ámbito donde se ubican las pruebas gráficas, específicamente la prueba del Dibujo de la Figura Humana (DFH) la cual es parte de este estudio y es utilizada en la evaluación del daño de la víctima como consecuencia de una agresión sexual. 
Dentro de las pruebas gráficas se encuentra el Dibujo de la Figura Humana (DFH), creada por Karen Machover en 1949. La base teórica que sustenta esta prueba señala que un individuo frente a la instrucción: "Dibuje una persona”, se halla obligado a dibujar partiendo de algunos referentes de apoyo. Las figuras externas son muy variadas en sus características corporales para presentarse espontáneamente en un dibujo, por lo que la persona debe realizar un proceso de selección de ciertos atributos. El individuo debe dibujar, consciente e inconscientemente, sobre su sistema completo de valores psíquicos, "siendo el cuerpo, o el propio yo, el punto más íntimo de referencia de cualquier actividad" (Goodenough, 1951, p. 4). La persona asocia ciertas sensaciones, percepciones y emociones con determinados órganos de su cuerpo. Estos órganos investidos son los que estructuran la imagen del cuerpo del individuo y los que, al ser percibidos, guían a la persona que está dibujando en la estructura específica y contenido del dibujo que realiza (Goodenough, 1951).

Mediante esta prueba el sujeto vehiculiza aspectos de la personalidad en relación a su autoconcepto e imagen corporal, comprendiendo que "el dibujo de una persona, al envolver la proyección de la imagen de un cuerpo, ofrece un medio natural de expresión de las necesidades y conflictos del cuerpo de uno" (Goodenough, 1951, p. 4).

El trabajo realizado por la autora es considerado pionero en la utilización del Dibujo de la Figura Humana para la proyección de la personalidad, obteniendo resultados favorables en la correlación entre la figura dibujada y la personalidad de quien lo hace. Debido a esto se ha extendido la aplicación de este instrumento a niños, adolescentes y adultos (Goodenough, 1951; Hammer, 2006).

Por otra parte, Machover (1949) descubrió que los aspectos estructurales y formales del dibujo como el tamaño, trazo y ubicación, se mantienen estables en el tiempo, por lo que se podría postular que las características reflejadas en el dibujo darían cuenta de la estructura de personalidad del sujeto. Koppitz agrega que el DFH también reflejaría "el estadio actual de desarrollo mental y las actitudes y preocupaciones 
en un momento dado, todo lo cual cambiará con el tiempo debido a la maduración y la experiencia” (Koppitz, 2006, p. 19), es decir, el DFH detectaría los cambios tanto evolutivos como emocionales en el sujeto.

El dibujo, al igual que el lenguaje hablado, puede analizarse de diversas formas o considerando distintos niveles. Por una parte, el dibujo puede evaluarse en términos de su estructura, es decir, considerando las características gráficas normalmente esperadas en cada etapa evolutiva. Y por otra, puede analizarse según su calidad, es decir, "por detalles poco comunes, omisiones o agregados" (Koppitz, 1991, p.2). Por último, se puede explorar el contenido de la producción gráfica, de manera de poder entender las claves del mensaje que el niño y/o adolescente está entregando.

Cabe señalar, además, que para un análisis adecuado de las producciones gráficas se deben considerar factores como el sexo, la madurez mental, la matriz cultural a la que el sujeto pertenezca y la facilitación o presión del medio ambiente (Machover, 1949). En relación al desarrollo de la habilidad gráfica hay diversos autores (Luque, Burt \& Goodenough, en Fernández, 1996; Koppitz, 1991) que plantean etapas o fases con características gráficas particulares para cada uno de éstos. El adolescente se ubica en los estadios finales del grafismo, pues se considera que ha completado su desarrollo en términos madurativos; no obstante, su grafismo tiene características diferentes a los dibujos adultos (Koppitz, 1991), ya que vivencia cambios emocionales propios de su grupo etário. Pese a los registros existentes, la prueba de DFH ha tenido dificultades en la incidencia de los resultados, debido a la poca claridad tanto en la definición de las variables o indicadores gráficos, como en los significados respecto a un mismo signo del DFH. Otra dificultad que se ha observado es que la mayoría de los autores que investigaban el DFH como prueba proyectiva minimizaban el aspecto evolutivo (Koppitz, 2006).

La prueba del Dibujo de la Figura Humana es parte de la batería psicodiagnóstica utilizada actualmente para la realización de peritajes en agresiones sexuales (Olea \& Rivera, 2007), específicamente para evaluar el daño que ha sufrido la víctima tras esta situación. A pesar de ser ampliamente ocupada, no existen investigaciones específicas que den cuenta de la validez 
de esta prueba para la evaluación del daño del adolescente agredido sexualmente, ni tampoco investigaciones que consideren la variable sexo de la víctima en sus análisis, por lo que la presente investigación cobra relevancia en este contexto, como un aporte a la validez de técnicas gráficas en hombres y mujeres adolescentes agredidos sexualmente.

A partir de la revisión teórica expuesta se plantea la siguiente problemática: ¿Existen indicadores gráficos diferenciales en la prueba del Dibujo de la Figura Humana en adolescentes hombres y mujeres, de 12 a 16 años, víctimas de agresiones sexuales? de esta pregunta se desprende el objetivo general de este estudio que es: determinar la existencia de indicadores gráficos diferenciales, en la prueba DFH, en adolescentes de 12 a 16 años, que hayan sufrido una agresión sexual crónica intrafamiliar o por parte de un conocido extrafamiliar, de aquellos de los que no se sospeche agresión sexual.

\section{Método}

Muestra

La muestra está compuesta por 76 adolescentes, entre 12 ańos y 16 años de ambos sexos. De éstos, un grupo corresponde al Grupo Agredido Sexualmente (GA), compuesto por 38 adolescentes, de ambos sexos, diagnosticados como víctimas de agresión sexual, y que asistan o hayan asistido al Centro de Atención a Víctimas de Atentados Sexuales (C.A.V.A.S.) o al Servicio Médico Legal (S.M.L.). Cabe señalar que para la conformación de la muestra se consideró indistintamente los diversos tipos de agresión sexual, ya que si bien en un comienzo se pretendía trabajar sólo con un tipo (abuso sexual o violación), debido a que éstos se asocian a determinadas consecuencias y síntomas específicos, no se encontró en las instituciones mencionadas el número de dibujos suficientes para la conformación de la muestra, lo que obligó a considerar ambos tipos de agresión sin distinción.

El segundo grupo fue denominado Grupo Control (GC), el cual está conformado por 38 adolescentes de similares características que el grupo anterior, salvo que en estos últimos no existe sospecha de agresión 
sexual. Dado que los adolescentes que asisten tanto al C.A.V.A.S. como al S.M.L pertenecen a un nivel socioeconómico bajo y medio-bajo (Avendaño, Grey, Osnovikoff, Oxman \& Vergara, 1993), se consideró también esta variable para la conformación del GC, el cual fue extraído de tres escuelas municipales pertenecientes a la comuna Pedro Aguirre Cerda, y que según información del SIMCE daban cuenta de esta condición socioeconómica.

Esta muestra es de tipo no probabilística o dirigida, ya que "la elección de los elementos no depende de la probabilidad, sino de causas relacionadas con las características de la investigación o de quien hace la muestra” (Baptista, Hernández \& Fernández, 2003, p. 305), en este caso la agresión sexual infantil.

El rango de edad específico considerado para esta investigación fue de 12 años 6 meses a 16 años 6 meses.

En el presente estudio se consideró para la conformación de la muestra la variable sexo, quedando cada grupo integrado por 27 casos de mujeres adolescentes (71\%) y 11 de hombres adolescentes (29\%). En la siguiente tabla se presenta la distribución de casos en relación al sexo en ambos grupos.

\begin{tabular}{|c|c|c|c|}
\hline $\begin{array}{c}\text { Sexo } \\
\text { Grupos }\end{array}$ & Mujeres & Hombres & Total \\
\hline GA & 27 & 11 & 38 \\
\hline GC & 27 & 11 & 38 \\
\hline Total & 54 & 22 & 76 \\
\hline
\end{tabular}

En un primer momento se consideró igualar el número de adolescentes hombres y mujeres en la muestra total; sin embargo, no se pudo llevar a cabo este objetivo por el número de dibujos disponibles en las instituciones. En el grupo agredido la obtención de casos de hombres fue inferior, lo cual podría explicarse por la menor cantidad de denuncias que éstos realizan (Servicio Nacional de Menores, 2008), resultando poco frecuentes en las instituciones señaladas anteriormente. 


\section{Instrumento}

Prueba del Dibujo de la Figura Humana.

\section{Procedimiento}

Para conformar la muestra del Grupo de adolescentes Agredidos Sexualmente (GA) que se incluyó en el presente estudio, se contactó a establecimientos que trabajan en el tema de los delitos sexuales. Por la posibilidad de acceder a la muestra escogió con el Centro de Atención a Víctimas de Atentados Sexuales (C.A.V.A.S.) y con la Unidad de Maltrato y Psiquiatría Infantil del Servicio Médico Legal, ubicados en las comunas de Providencia e Independencia, respectivamente.

Luego de este contacto previo, los Centros dieron acceso al DFH de los adolescentes que cumplieran los requisitos exigidos para la muestra.

En paralelo, se procedió a conformar el Grupo Control (GC), que estuvo compuesto por adolescentes sin sospecha de agresión sexual ni otra patología o trastorno emocional, con características similares al GA (nivel educacional, edad y nivel socioeconómico). Para determinar de manera eficiente los sujetos del GC más idóneos para la muestra, se solicitó a los apoderados de los alumnos de los cursos seleccionados contestar un breve cuestionario mediante el cual se indagaba la presencia de enfermedades médicas de importancia, problemas emocionales actuales o anteriores, que pudieran haber ocasionado un trastorno por estrés postraumático y tratamientos psiquiátricos y/o psicológicos previos, con el respectivo diagnóstico. Además, se realizaron entrevistas con el profesor jefe del adolescente, en la cual se indagó, a través de un cuestionario, factores de riesgo en la familia y/o entorno de los alumnos, presencia de síntomas y conductas relacionadas con un trastorno por estrés postraumático, por agresión sexual y/o maltrato físico. Ahora bien, los adolescentes que presentaron alguno de los factores mencionados anteriormente se excluyeron de la muestra del GC.

Para el análisis de los DFH se generó una lista con las definiciones operacionales de ciertos indicadores gráficos a considerar en la evaluación, 
los que fueron recogidos de investigaciones anteriores centradas en la temática de agresiones sexuales, adolescencia y otras. Entre éstas se encuentran los aportes realizados por Machover (1949); Goodenough (1951); Koppitz (2006); Buck (1995); Barilari; Beigbeder \& Colombo (2000); Cuadra \& Ureta (2005); Girardi \& Pool (2005) y Miranda \& Sanza (2004), centrados tanto en las definiciones operacionales de los indicadores gráficos como en la asociación de éstos con determinadas vivencias emocionales.

La pauta de indicadores gráficos utilizados para el análisis del Dibujo de la Figura Humana fue construida por las investigadoras, basadas en los autores ya mencionados, siendo asesoradas en este proceso por la psicóloga Ruth Weinstein, experta en la temática de Pruebas Proyectivas.

Para efectuar un análisis objetivo y confiable se decidió analizar de forma conjunta los DFH de los adolescentes de ambos grupos (GA y GC), a partir de un análisis a ciegas, es decir, "sin que el intérprete supiera nada más que el sexo y la edad del examinado" (Hammer, 2006, p. 157), contando con la asesoría de un Juez experto. En el presente estudio participó la Psicóloga Ruth Weinstein como Jueza experta, la cual fue elegida para este fin por su calidad de especialista en Pruebas Gráficas y por el acceso a ella por parte de las investigadoras.

\section{Resultados}

En la presente investigación se trabajó con 130 indicadores gráficos, los cuales fueron dicotomizados según la ausencia o presencia de cada uno de éstos, debido a que el número total de casos que conformaron la muestra fue de 76. De esta forma se evitó una excesiva dispersión de los casos en las categorías de cada indicador gráfico.

Considerando que las variables en estudio son datos nominales y que la investigación no tiene supuestos acerca de la distribución poblacional, se realizó un análisis no paramétrico de los datos (Baptista et al., 2003).

Para evaluar las hipótesis acerca de la relación entre los distintos grupos de estudio, se aplicó la prueba estadística de chi cuadrado, la cual se calculó a través de una tabla de contingencia de dos dimensiones $(2 \times 2)$, 
donde cada dimensión comprendió una variable y ésta a su vez incluyó dos categorías. Debido a que se utilizó la tabla antes mencionada, se consideró chi cuadrado por corrección por continuidad, con el fin de obtener el nivel de significación; si éste es menor a 0,05 no habría relación entre los grupos de estudio (Baptista et al., 2003). Para la presente investigación se consideró un grado de libertad.

A continuación se presenta el análisis de los datos:

Resultado comparativo entre mujeres y hombres pertenecientes al "Grupo Agredido sexualmente":

En relación a los 130 indicadores gráficos, hubo cinco indicadores estadísticamente significativos al correlacionar mujeres y hombres del GA, que son:

\begin{tabular}{|l|c|c|c|}
\hline \multirow{2}{*}{ Indicador Gráfico } & \multicolumn{2}{|c|}{ Presencia del Indicador } & \multirow{2}{*}{$\begin{array}{c}\text { Nivel de } \\
\text { Significación }\end{array}$} \\
\cline { 2 - 3 } & Mujeres GA & $\begin{array}{c}\text { Hombres } \\
\text { GA }\end{array}$ & \\
\hline $\begin{array}{l}\text { 1. Ubicación vertical } \\
\text { zona media }\end{array}$ & $\mathbf{1 3 ( 4 8 \% )}$ & $1(9 \%)$ & $\mathbf{0 , 0 3}$ \\
\hline $\begin{array}{l}\text { 2. Ubicación vertical } \\
\text { zona inferior }\end{array}$ & $8(30 \%)$ & $\mathbf{9 ( 8 2 \% )}$ & $\mathbf{0 , 0 1}$ \\
\hline $\begin{array}{l}\text { 3. Línea media } \\
\text { horizontal }\end{array}$ & $18(67 \%)$ & $\mathbf{1 1 ( 1 0 0 \% )}$ & $\mathbf{0 , 0 4}$ \\
\hline $\begin{array}{l}\text { 4. Énfasis en el pelo } \\
\text { 5. Dibujo ambiguo }\end{array}$ & $\mathbf{2 1 ( 7 8 \% )}$ & $4(36 \%)$ & $\mathbf{0 , 0 2}$ \\
\hline
\end{tabular}

Considerando lo anterior, se podría mencionar que el GA funcionaría como dos grupos disímiles en cinco indicadores gráficos si se considera la variable sexo. Es decir, los hombres y mujeres del GA 
presentarían diferencias significativas en estos indicadores, al realizar el DFH. Sin embargo, funcionaría como un grupo homogéneo en 125 indicadores gráficos, es decir, no habría diferencias significativas entre hombres y mujeres del GA en estos indicadores al realizar el DFH.

Resultado comparativo entre mujeres y hombres pertenecientes al "Grupo Control":

En relación a los 130 indicadores gráficos, hubo nueve indicadores estadísticamente significativos al correlacionar mujeres y hombres del GC. Éstos son:

\begin{tabular}{|c|c|c|c|}
\hline \multirow[t]{2}{*}{ Indicador Gráfico } & \multicolumn{2}{|c|}{ Presencia del Indicador } & \multirow{2}{*}{$\begin{array}{c}\text { Nivel de } \\
\text { Significación }\end{array}$} \\
\hline & $\begin{array}{c}\text { Mujeres } \\
\text { GC }\end{array}$ & $\begin{array}{c}\text { Hombres } \\
\text { GC }\end{array}$ & \\
\hline 1. Trazo reforzado & $7(26 \%)$ & $8(73 \%)$ & 0,01 \\
\hline 2. Presión fuerte & $10(37 \%)$ & $9(82 \%)$ & 0,03 \\
\hline 3. Ojos grandes & $6(22 \%)$ & $7(64 \%)$ & 0,02 \\
\hline 4. Énfasis en los ojos & $11(41 \%)$ & $9(82 \%)$ & 0,05 \\
\hline 5. Énfasis en la nariz & $2(7 \%)$ & $6(55 \%)$ & 0,00 \\
\hline 6. Nariz grande & $2(7 \%)$ & $5(46 \%)$ & 0,01 \\
\hline 7. Énfasis en las orejas & $7(26 \%)$ & $8(73 \%)$ & 0,01 \\
\hline $\begin{array}{l}\text { 8. Símbolos fálicos } \\
\text { agresivos }\end{array}$ & $0(0 \%)$ & $3(27 \%)$ & 0,02 \\
\hline 9. Borradura de boca & $10(37 \%)$ & $0(0 \%)$ & 0,04 \\
\hline 10. Cierre del pantalón* & $2(7 \%)$ & $6(55 \%)$ & 0,00 \\
\hline 11. Otro símbolo genital* & $1(4 \%)$ & $6(55 \%)$ & 0,00 \\
\hline
\end{tabular}

* Estos indicadores gráficos resultaron emergentes dentro del presente estudio. 
Tras lo expuesto podríamos concluir que el GC funcionaría como dos grupos disímiles en nueve indicadores gráficos considerando la variable sexo, es decir, los hombres y mujeres del GA presentarían diferencias significativas en estos indicadores, al realizar el DFH. Sin embargo, funcionaría como un grupo homogéneo en 121 indicadores gráficos al realizar el DFH.

Resultado comparativo entre el "Grupo agredido sexualmente" y el "Grupo Control":

En este apartado se presentarán los resultados de la comparación del GA y el GC en relación a la presencia o ausencia de cada uno de los indicadores gráficos. Cabe mencionar que la variable sexo será considerada dentro del análisis de los cinco indicadores gráficos que resultaron ser significativos en el Resultado comparativo entre mujeres y hombres pertenecientes al $G A$, y los nueve indicadores que resultaron ser significativos en el Resultado comparativo entre mujeres y hombres pertenecientes al GC. Es decir, se trabajará con el supuesto de que existirían diferencias considerando el sexo de los sujetos en relación a estos 14 indicadores gráficos, al realizar el DFH.

El nivel de significación de los 116 indicadores gráficos restantes será evaluado comparando el total del GA y el total del GC, estableciendo que las mujeres y hombres de cada grupo responderían de manera similar frente a estos indicadores gráficos, al realizar el DFH.

Resultado comparativo entre mujeres y hombres pertenecientes al "Grupo Control" y, mujeres y hombres pertenecientes al "Grupo Agredido":

Al comparar el GA con el GC en relación a los 116 indicadores gráficos que no tuvieron diferencias en relación al sexo, se observa que 14 resultaron ser significativos. Éstos son: 


\begin{tabular}{|c|c|c|c|}
\hline \multirow{2}{*}{ Indicador Gráfico } & \multicolumn{2}{|c|}{ Presencia del Indicador } & \multirow{2}{*}{$\begin{array}{c}\text { Nivel de } \\
\text { Significación }\end{array}$} \\
\hline & GC & GA & \\
\hline 1. Trazo circular & $3(8 \%)$ & $13(34 \%)$ & 0,01 \\
\hline 2. Trazo fragmentado & $31(82 \%)$ & $21(55 \%)$ & 0,03 \\
\hline $\begin{array}{l}\text { 3. Énfasis en la línea } \\
\text { media horizontal }\end{array}$ & $29(76 \%)$ & $14(37 \%)$ & 0,00 \\
\hline 4. Ojos vacíos & $1(3 \%)$ & $10(27 \%)$ & 0,01 \\
\hline 5. Cejas y/o pestañas & $28(74 \%)$ & $18(47 \%)$ & 0,04 \\
\hline 6. Bolsillos & $18(47 \%)$ & $6(16 \%)$ & 0,01 \\
\hline 7. Símbolos fálicos & $30(79 \%)$ & $18(47 \%)$ & 0,01 \\
\hline 8. Borradura de cuello & $16(42 \%)$ & $6(16 \%)$ & 0,02 \\
\hline 9. Borradura de tronco & $14(37 \%)$ & $3(8 \%)$ & 0,01 \\
\hline 10. Borradura de brazos & $26(68 \%)$ & $12(32 \%)$ & 0,00 \\
\hline 11. Borradura de manos & $20(53 \%)$ & $5(13 \%)$ & $\mathbf{0 , 0 0}$ \\
\hline 12. Borradura de piernas & $19(50 \%)$ & $4(11 \%)$ & 0,00 \\
\hline $\begin{array}{l}\text { 13. Borradura de zona } \\
\text { genital }\end{array}$ & $6(16 \%)$ & $0(0 \%)$ & 0,03 \\
\hline 14. Sombreado de brazos & $9(24 \%)$ & $2(5 \%)$ & 0,05 \\
\hline
\end{tabular}

Resultado comparativo entre adolescentes hombres pertenecientes al "Grupo agredido sexualmente" y adolescentes hombres pertenecientes al "Grupo control":

Al comparar los hombres del GA con los hombres del GC, en relación a los 14 indicadores gráficos que tuvieron diferencias en relación al sexo, se observa que dos resultaron ser significativos. Éstos son: 


\begin{tabular}{|c|c|c|c|}
\hline \multirow[b]{2}{*}{ Indicador Gráfico } & \multicolumn{2}{|c|}{ Presencia del Indicador } & \multirow{2}{*}{$\begin{array}{c}\text { Nivel de } \\
\text { Significación }\end{array}$} \\
\hline & $\begin{array}{c}\text { Hombres } \\
\text { GC }\end{array}$ & $\begin{array}{c}\text { Hombres } \\
\text { GA }\end{array}$ & \\
\hline $\begin{array}{l}\text { 1. Ubicación vertical zona } \\
\text { inferior. }\end{array}$ & $2(18 \%)$ & $9(82 \%)$ & 0,01 \\
\hline 2. Ojos grandes. & $7(64 \%)$ & $1(9 \%)$ & 0,02 \\
\hline 3. Simbolismo genital.* & $6(55 \%)$ & $0(0 \%)$ & $\mathbf{0 , 0 1}$ \\
\hline
\end{tabular}

* Este indicador gráfico fue un emergente dentro de la presente investigación.

Resultado comparativo entre mujeres adolescentes pertenecientes al "Grupo agredido sexualmente" y mujeres adolescentes pertenecientes al "Grupo control":

Al comparar las mujeres del GA con las mujeres del GC, en relación a los 14 indicadores gráficos que tuvieron diferencias en relación al sexo, se observa que el indicador Línea media horizontal resultó ser significativo, siendo más frecuente en los dibujos de las mujeres del GC.

\begin{tabular}{|c|c|c|c|}
\hline \multirow{2}{*}{ Indicador Gráfico } & \multicolumn{2}{|c|}{ Presencia del Indicador } & \multirow{2}{*}{$\begin{array}{c}\text { Nivel de } \\
\text { Significación }\end{array}$} \\
\cline { 2 - 3 } & $\begin{array}{c}\text { Mujeres } \\
\text { GC }\end{array}$ & $\begin{array}{c}\text { Mujeres } \\
\text { GA }\end{array}$ & \\
\hline 1. Línea media horizontal & $\mathbf{2 5 ( 9 3 \% )}$ & $18(67 \%)$ & $\mathbf{0 , 0 4}$ \\
\hline
\end{tabular}

Discusión

Tras el análisis de los resultados, en primer lugar se puede concluir que la prueba DFH discrimina entre adolescentes de ambos sexos de 12 a 16 años que hayan sufrido una agresión sexual crónica intrafamiliar o por parte de un conocido extrafamiliar, de los adolescentes que no se sospeche hayan sido víctima de dicha agresión. Lo que daría cuenta de esta 
afirmación son los 17 indicadores gráficos diferenciales en la prueba DFH entre adolescentes pertenecientes al GA y al GC. Según su frecuencia se distribuyen de la siguiente manera:

\begin{tabular}{|c|c|}
\hline Más frecuente en GC & Más frecuente en GA \\
\hline Trazo fragmentado & Trazo circular \\
\hline Línea media horizontal & Ubicación vertical zona inferior \\
\hline Énfasis en línea media horizontal & Ojos vacíos \\
\hline Cejas y/o pestańas & \\
\hline Ojos grandes & \\
\hline Bolsillos & \\
\hline Símbolos fálicos & \\
\hline $\begin{array}{c}\text { Borradura de cuello, tronco, } \\
\text { brazos, manos, piernas y zona } \\
\text { genital }\end{array}$ & \\
\hline Sombreado de brazos & \\
\hline
\end{tabular}

La alta significación de los tres indicadores gráficos asociados al GA podría estar relacionada con el daño emocional de los adolescentes agredidos sexualmente. De estos indicadores, Trazo circular ha sido relacionado con características de dependencia (Hammer, 2006; Machover, 1949). En este sentido es preciso señalar que uno de los efectos psicológicos como consecuencia de una agresión sexual sería una alteración vincular, la cual se podría presentar de dos maneras: como una desconfianza excesiva, o como una reacción contraria de dependencia extrema, necesitando establecer relaciones intensas de confianza y seguridad (Policía de Investigaciones de Chile, 2003).

Por lo anterior, se podría pensar que los y las adolescentes agredidos sexualmente, que reaccionan de forma más dependiente, tendrían una tendencia a realizar el DFH con predominio de líneas circulares. Por otra parte, otras investigaciones (Hammer, 2006) manifiestan que la presencia de este indicador se esperaría en niños sin sospecha de agresión sexual, ya 
que éstos son normalmente inmaduros y dependientes debido a la etapa evolutiva que atraviesan; sin embargo, no se esperaría en adolescentes estables emocionalmente, ya que éstos han desarrollado más independencia en relación a su entorno.

A su vez, el indicador gráfico Ojos vacíos se asociaría a una tendencia del sujeto a evitar estímulos visuales displacenteros (Buck, 1995), lo cual podría relacionarse con el mecanismo defensivo de disociación utilizado frecuentemente por los adolescentes agredidos sexualmente, quienes buscarían eludir o evitar cualquier evocación o recuerdo de la experiencia traumática debido a la alta carga emocional negativa que esto conlleva (Barudy, 1998). Este mecanismo defensivo fue consignado en las fichas clínicas de los pacientes adolescentes del C.A.V.A.S., por lo cual se podría pensar que estos jóvenes tendrían una tendencia a realizar los ojos del DFH sin la presencia de pupilas.

Por otra parte, el indicador gráfico Ubicación vertical zona inferior, significativo en el GA, estaría asociado a inseguridad, temor a perder contacto con la realidad por exceso de emotividad y ánimo depresivo (Buck, 1995). En este sentido, los adolescentes agredidos sexualmente presentarían de forma reiterada síntomas asociados a la depresión (Intebi, 1998; De Corral \& Echeburúa, 2006; Policía de Investigaciones de Chile, 2003), los cuales se originarían por una baja autoestima, debido a la internalización de una imagen de sí mismo deteriorada por los efectos traumáticos de la experiencia abusiva (Barudy, 1998). Estos aspectos se observaron en las fichas clínicas de pacientes del C.A.V.A.S. Cabe señalar que este indicador se observó con mayor frecuencia en los hombres adolescentes agredidos sexualmente (82\%) en comparación a las mujeres de las mismas características (30\%). Esto podría estar asociado a que los hombres estarían más expuestos a distintos tipos de maltrato físico, ya que opondrían más resistencia que las mujeres frente a una agresión sexual (Servicio Nacional de Menores, 2008), generándose un mayor daño en su cuerpo, lo que conllevaría a una internalización de una imagen de sí mismo más deteriorada. Además, se podría pensar que la estigmatización como consecuencia de una agresión sexual es mayor en los adolescentes hombres, ya que se asocia a fantasías relacionadas con la homosexualidad. 
Ambos elementos podrían llevar a una intensificación de sintomatología depresiva en los hombres agredidos sexualmente.

En relación a los 14 indicadores gráficos asociados al GC (Trazo fragmentado, Linea media horizontal, Énfasis en línea media horizontal, Cejas y/o pestañas, Ojos grandes, Bolsillos, Simbolos fálicos, Borradura de cuello, tronco, brazos, manos, piernas y zona genital y Sombreado de brazos), éstos podrían estar relacionados con dimensiones propias de la adolescencia, como son la sexualización y erotización, que dan cuenta de cambios físicos del cuerpo y, con ello, el despertar sexual (Florenzano, 2005), además de una preocupación por lo externo y los detalles (Koppitz, 1991), también características de este grupo etário.

A pesar de que los indicadores antes mencionados estarían asociados a la adolescencia, se presentarían con una menor frecuencia en el grupo de adolescentes agredidos sexualmente. Esto podría relacionarse con los efectos que la agresión sexual ocasionaría en el continuo vital del adolescente vulnerado. Es decir, el adolescente agredido, al depositar gran parte de sus energías en la elaboración de las vivencias y experiencias asociadas a la agresión sexual (Barudy, 1998), tendría menos energía para invertir en la elaboración de las vivencias propias de su etapa evolutiva, lo cual se reflejaría en su producción gráfica.

Los indicadores gráficos Cejas y pestañas, Ojos grandes, Simbolos fálicos, Linea media y Énfasis en la línea media horizontal, podrían dar cuenta de los procesos de sexualización y erotización propios de la adolescencia. El adolescente, al dibujar la Figura Humana con Cejas, pestañas y/u Ojos grandes manifestaría su preocupación por resaltar ciertas características faciales en el dibujo, lo cual podría relacionarse con un "Querer ser visto y ver-se". Cabe destacar, además, que los hombres del GC tendieron a dibujar, con mayor frecuencia que las mujeres del mismo grupo, dibujos con ojos de gran tamaño, lo cual podría relacionarse con que los hombres adolescentes estarían más motivados a "querer ver", lo que daría cuenta de un deseo por controlar activamente su entorno, concordante con un rol más activo de los hombres, siendo éste adquirido mediante un aprendizaje social (Servicio Nacional de Menores, 2008). 
Por otra parte, que estos dos indicadores gráficos se presenten con menor frecuencia en los dibujos de adolescentes agredidos sexualmente, podría revelar una tendencia de éstos a esconder u omitir las características faciales (cejas, pestañas y ojos grandes) que pudieran ser atractivas para un Otro, el cual pudiese resultarles amenazante, siendo esto propio de la sintomatología abusiva (Policía de Investigaciones de Chile, 2003). Por otro lado, la escasez de ojos grandes, tanto en los dibujos realizados por los hombres como por las mujeres adolescentes del GA, podría relacionarse con una tendencia de éstos a desvincularse de su entorno y de sí mismos (dejar de ver), pues tendrían cierto temor a contactarse con experiencias y vivencias dolorosas. Es decir, el adolescente "No quiere ser visto ni ver-se".

La presencia de símbolos fálicos en el DFH realizado por adolescentes denotaría cierta ansiedad con respecto a la sexualidad (Machover, 1949). En este sentido, dibujar la línea media y/o resaltarla, se relacionaría con un gran interés y preocupación por la temática de la sexualidad, tendiendo a dibujar elementos en la figura humana que marcan o señalan la línea media horizontal (ejemplo: un cinturón) como forma de regular los impulsos sexuales, ya que existiría un conflicto en su control y expresión (Buck, 1995).

Los indicadores Simbolos fálicos y Énfasis en la línea media horizontal se presentarían con menor frecuencia en los dibujos de los adolescentes agredidos, lo cual manifestaría una tendencia a anular la sexualidad en las producciones gráficas, incorporándola de forma inapropiada o disfuncional, lo cual sería una consecuencia de la transgresión a sus límites corporales y de la vulneración de su sexualidad por un Otro (Policía de Investigaciones de Chile, 2003).

Por otro lado, el indicador Linea media horizontal no presentaría diferencias en relación a los hombres adolescentes del GA y del GC, encontrándose en el $100 \%$ de los casos de ambos grupos. Sin embargo, podría tener connotaciones distintas para cada uno; así, en los hombres adolescentes del GA reflejaría una necesidad de tener el control, en la medida que regula y reprime sus impulsos sexuales. En cambio para los hombres adolescentes del GC se asociaría una preocupación e interés por el cuerpo nuevo que crece, propios de su desarrollo evolutivo. En relación 
al género femenino, existirían diferencias entre ambos grupos, debido a que se presentaría con menor frecuencia en las mujeres adolescentes del GA, en comparación con las mujeres adolescentes del GC, lo que daría cuenta, de que al intervenir la variable agresión sexual, las mujeres adolescentes del GA tenderían a disminuir la regulación y represión de sus impulsos sexuales, lo cual podría relacionarse con un comportamiento sexual precoz y desinhibido. Todo lo expuesto se comprende desde el concepto de "Vampirización" (Barudy, 1998), en el cual la adolescente tiene conductas erotizadas como una forma de recuperar el dominio de sí misma, dominio que ha perdido debido a la sexualidad traumática, inapropiada e incontrolable que ha instalado la dinámica abusiva.

Los indicadores gráficos Trazo fragmentado y Borraduras darían cuenta de una dimensión de la adolescencia relacionada con lo estético, es decir, la preocupación por lo externo y los detalles. En este sentido, estos indicadores harían referencia al cuidado con que el adolescente realiza el dibujo, poniendo gran interés en los elementos estéticos de éste (Koppitz, 1991), como la línea (Trazo fragmentado) y la forma (Borradura). Si bien estos indicadores gráficos son frecuentemente relacionados con ansiedad y angustia (Buck, 1994; Hammer, 2006; Koppitz, 2006; Machover, 1949), se observó durante la aplicación de la Prueba del DFH en las escuelas con las que se trabajó, que los adolescentes utilizaban el predominio de líneas fragmentadas en el dibujo como forma de resaltar ciertas características de éste, dándole una mejor calidad visual. En este mismo sentido, se observó durante la aplicación que los adolescentes borraban ciertas partes del dibujo como forma de mejorar la producción gráfica, logrando este objetivo en la mayoría de los casos. Por último, se puede mencionar que las condiciones contextuales (lugar acogedor, sin límite de tiempo, escaso ruido, etc.) podrían haber disminuido los montos de angustia inicial de los adolescentes, los cuales realizaban el dibujo de manera tranquila y prolija. Que estos dos indicadores mencionados aparecieran con menor frecuencia en los dibujos de los adolescentes agredidos sexualmente podría relacionarse con las condiciones en las cuales se efectúa este dibujo. Al realizar la aplicación de la Prueba del DFH en un contexto de peritaje y/o en las etapas iniciales de un proceso psicoterapéutico, podría llevar a los adolescentes a produccio- 
nes gráficas en las cuales no borran, tal vez asociado al deseo del adolescente agredido sexualmente de terminar el dibujo de forma rápida, debido al alto monto de angustia que le genera la situación de evaluación, ya que ésta implicaría recordar el hecho traumático y establecer una relación vincular con un otro desconocido, llevándolo a despreocuparse de los elementos estéticos del dibujo. En este sentido, se observó que la mayoría de los protocolos de pacientes del C.A.V.A.S. dan cuenta de síntomas de angustia y ansiedad ante las primeras situaciones de entrevista y a la dificultad de establecer un vínculo con el adolescente por parte del perito o psicoterapeuta.

Ahora bien, el indicador gráfico Bolsillos estaría asociado a un conflicto entre la dependencia y el deseo de autonomía (Machover, 1949), propio de la etapa de la adolescencia, en donde los jóvenes buscan diferenciarse de sus figuras parentales como forma de construir una identidad propia. Este indicador se presenta con menor frecuencia en los adolescentes agredidos sexualmente, quienes se muestran más dependientes, debido a una falla en la manera de vincularse, la cual estaría relacionada con la traición que sienten, por un lado, del adulto en el que depositaban su confianza y los agredió, y por otro, del adulto que no los resguardó para evitar que la agresión sexual se llevase a cabo (Policía de Investigaciones de Chile, 2003).

Por último, el indicador gráfico Sombreado de brazos ha sido asociado a ansiedad con respecto al contacto y a la adaptación social (Koppitz, 2006; Machover, 1949), lo cual es propio de la etapa de la adolescencia, en donde el sujeto se siente inadecuado y extraño debido a los cambios emocionales y físicos que va experimentando, viéndose dificultoso el adaptarse a lo que lo rodea, lo que finalmente le produce ansiedad y angustia. Este indicador se presentó con menor frecuencia en los dibujos de adolescentes agredidos sexualmente, lo que podría estar relacionado con una dificultad para contactarse con los demás, utilizando mecanismos de defensa que los llevan a reducir sus contactos con el mundo exterior. Este estado correspondería a la anestesia psíquica y emocional, descrita en el Manual Diagnóstico y Estadístico de los Trastornos Mentales (DSM-IV) o al estado de evitación e insensibilidad descrito por Ammerman y Hersen (Barudy, 1998), encontrándose dentro de sus síntomas: aislamiento social, reticencia a salir a algún lugar determinado, desinterés por actividades que antes eran atractivas, entre otros. 
Por último, considerando los resultados del presente estudio, se podría afirmar que la Prueba DFH discrimina entre los hombres adolescentes de 12 a 16 años que hayan sufrido una agresión sexual crónica intrafamiliar o por parte de un conocido extrafamiliar, y mujeres adolescentes de las mismas características. Lo que sustenta dicha afirmación es la presencia de cinco indicadores gráficos diferenciales en la prueba DFH entre adolescentes hombres y mujeres pertenecientes al GA, los que se presentan a continuación:

\begin{tabular}{|c|c|}
\hline $\begin{array}{c}\text { Más frecuente en } \\
\text { hombres del GA }\end{array}$ & $\begin{array}{c}\text { Más frecuente en } \\
\text { mujeres del GA }\end{array}$ \\
\hline Ubicación vertical zona inferior & Ubicación vertical zona media \\
\hline Línea media horizontal & Énfasis en el pelo \\
\hline Dibujo ambiguo & \\
\hline
\end{tabular}

Asimismo, el análisis de los datos obtenidos refleja que la Prueba DFH discriminará entre hombres y mujeres adolescentes de 12 a 16 años, que no se sospeche que hayan sufrido una agresión sexual, maltrato o presencia de factores estresores importantes. La información que corrobora esta afirmación son los 9 indicadores gráficos diferenciales en la prueba DFH entre adolescentes hombres y mujeres pertenecientes al GC, los que se presentan a continuación:

\begin{tabular}{|c|c|}
\hline $\begin{array}{c}\text { Más frecuente en } \\
\text { hombres del GC }\end{array}$ & $\begin{array}{c}\text { Más frecuente en } \\
\text { mujeres del GC }\end{array}$ \\
\hline Trazo reforzado & Borradura de boca \\
\hline Presión fuerte & \\
\hline Énfasis en los ojos & \\
\hline Ojos grandes & \\
\hline Énfasis en la nariz & \\
\hline Nariz grande & \\
\hline Énfasis en las orejas & \\
\hline Símbolos fálicos agresivos & \\
\hline
\end{tabular}


Por lo tanto, se podría afirmar que los indicadores gráficos diferenciales de adolescentes, hombres y mujeres de 12 a 16 años, que hayan sufrido una agresión sexual crónica intrafamiliar o por parte de un conocido extrafamiliar, serán distintos de los indicadores gráficos diferenciales de adolescentes, hombres y mujeres de 12 a 16 años, que no se sospeche hayan sufrido dicha agresión. En este sentido, esta afirmación daría cuenta de una tendencia de los adolescentes hombres y mujeres a realizar el DFH de manera distinta cuando interviene la variable agresión sexual, es decir, la situación abusiva afectaría de forma diferente a los hombres y mujeres agredidos sexualmente.

Los nueve indicadores gráficos diferenciales entre adolescentes hombres y mujeres del GC darían cuenta de procesos y conflictos relacionados con su etapa evolutiva y con la variable sexo. El hecho de que estos nueve indicadores no resultaran significativos al comparar hombres y mujeres del GA podría manifestar, como ya se mencionó, un alejamiento de este grupo de las temáticas propias de la adolescencia, debido a que estarían vivenciando distintos procesos, canalizarían sus energías en la resolución de conflictos en relación con la agresión sexual (Barudy, 1998). Cabe destacar que la elaboración de dicha agresión sería distinta considerando la variable género para este grupo, existiendo cinco indicadores gráficos diferenciales entre adolescentes hombres y mujeres del grupo agredido.

Por último, se considera que todos las apreciaciones expuestas podrían proponer a la prueba del Dibujo de la Figura Humana como un instrumento potencial para seguir formando parte de una batería psicodiagnóstica, con el fin de evaluar el daño emocional asociado a una agresión sexual, siendo el presente estudio un aporte para la fundamentación de las pruebas proyectivas gráficas en las evaluaciones psicológicas de adolescentes agredidos sexualmente, tanto en el área clínica como judicial.

Se considera relevante continuar el desarrollo de estudios que permitan ampliar y validar conocimientos con respecto a las pruebas proyectivas gráficas en evaluación de daño en agresión sexual. Esto cobra mayor valor si se considera que la mayoría de los niños y adolescentes 
agredidos sexualmente presenta dificultades en poner en palabras sus sentimientos y expresar emociones, por lo traumático del hecho vivido, siendo las producciones gráficas una alternativa para acceder a su mundo interno, asumiendo que las pruebas gráficas no se presentan como un instrumento que por sí solo pueda determinar tajantemente la existencia de daño asociado a una agresión sexual.

Como futuras líneas de investigación se plantea la reproducción de este estudio con distintos grupos etários, con el fin de definir características específicas en cada uno de ellos y generar estimaciones del tipo y grado de daño en relación a la agresión sexual, además de determinar en qué grupo etário la prueba DFH podría tener un mayor poder diagnóstico. Asimismo, se propone trabajar con un mayor número de casos, para que los resultados sean más significativos y, por ende, posibles de extrapolar a la población.

Por último, se considera relevante incorporar, en investigaciones futuras, grupos de estudios que presenten distintas secuelas de estrés posttraumático, con el fin de determinar indicadores gráficos específicos para cada tipo de vulneración.

\section{Referencias}

Alhambra, C., Huerta, C., Miranda, M., Navarro, C. \& Prado, C. (2004, Diciembre). Pericias Psicológicas en víctimas de delitos sexuales: metodología de evaluación clínico-pericial desarrollada en el CAVAS metropolitano. Documento presentado en el XVI Congreso Internacional de Ciencias Forenses, Santiago, Chile.

Avendaño, C., Grey, N., Osnovikoff, I., Oxman, V. \& Vergara, J. (1993) Violencia Sexual en Chile. Algunos indicadores relevantes. Recuperado el 13 de abril del año 2008 de http://www.pasa.cl/ index.php?option $=$ com_docman $\&$ task $=$ cat_view $\&$ gid $=52 \& \mathrm{dir}=\mathrm{D}$ ESC\&order $=$ name\&limit $=10$ \&limitstart $=10$

Bain, C. \& Yáñez, M.A. (2002). Carencias que duelen: Servicios para mujeres y niñas afectadas por violencia sexual en Chile. Santiago: LOM.

Baptista, P., Hernández, R. \& Fernández, C. (2003). Metodología de la Investigación. México: Mc Graw-Hill Interamericana. 
Barilari, Z. Beigbeder, C. \& Colombo, R. (2000). Indicadores de abuso y maltrato infantil en la prueba gráfica persona bajo la lluvia. Buenos Aires: Paidós.

Barudy, J. (1998). El dolor invisible en la infancia: una lectura ecosistémica del maltrato infantil. Barcelona: Paidós Ibérica.

Buck, J. (1995). Manual y guia de interpretación de la técnica de dibujo proyectivo. HTP. México. D.F.: El Manual Moderno. (Orig. 1992).

Carvacho, P., Morales, G. \& Salman, V. (1998). Evaluación del desarrollo psicosexual en niñas entre los 6 y 8 años en la Región Metropolitana, que han sido victimas de abuso sexual. Tesis de licenciatura no publicada. Universidad Diego Portales. Santiago, Chile.

Corsi, J. (1994). Violencia familiar: Una mirada interdisciplinaria sobre un grave problema social. Buenos Aires: Paidós.

Cuadra, A. \& Ureta, P. (2005). Estudio descriptivo comparativo del test H-T-P entre adolescentes victimas de agresiones sexuales y adolescentes sin sospecha de agresión sexual. Tesis de título no publicada. Universidad de Tarapacá, Arica, Chile.

Chía, E., Fernández, H., Navarro, J., Salgado, R. \& Valdés, A. (2005). Protocolo para la evaluación psicológica pericial de delitos sexuales contra niños, niñas y adolescentes. Santiago: LOM.

De Corral, P. \& Echeburúa, E. (2006). Secuelas emocionales en víctimas de abuso sexual en la infancia. Cuadernos de Medicina Forense, 12, 75-82.

Fernández, M. (1996). El dibujo de la figura humana. Aspectos psicométricos $y$ proyectivos en el proceso psicodiagnóstico. Buenos Aires: Psicoteca.

Florenzano, R. (2005). Desarrollo y salud integral del adolescente. Diploma a distancia impartido por la Pontificia Universidad Católica de Chile, Santiago, Chile. Recuperado el 31 de octubre del año 2008 de: http://escuela.med.puc.cl/paginas/OPS/Curso/Lecciones/ Leccion13/M3L13Leccion.html

Girardi, K. \& Pool, A. (2005). Evaluación de indicadores gráficos asociados a agresiones sexuales en la prueba persona bajo la lluvia en niños victimizados sexualmente de 9 a 11 años de edad. Un estudio descriptivocomparativo. Memoria de título de Psicólogo no publicada. Universidad de Chile, Santiago, Chile.

Goodenough, F. (1951). Test de inteligencia infantil: por medio del dibujo de la figura humana. Buenos Aires: Paidós.

Hammer, E. (2006). Test proyectivos gráficos. Buenos Aires: Paidós.

Intebi, I. (1998). Abuso sexual infantil: En las mejores familias. Barcelona: Gránica.

Koppitz, E. (2006). El dibujo de la figura humana en los niños. (12a ed.) Buenos Aires: Guadalupe. (Orig. 1974). 
Koppitz, E. (1991). Evaluación psicológica de los dibujos de la figura humana por alumnos de enseñanza media. México, D.F.: El Manual Moderno. (Orig. 1984).

Llanos, M.T. \& Sinclair, A (2001). Terapia de Reparación en Víctimas de Abuso Sexual. Aspectos Fundamentales. Psykhé, 10(2), 53-70.

Machover, K. (1949). Proyección de la personalidad en el dibujo de la figura humana. Habana: Cultural.

Maffioletti, F. \& Salinas, M. (2005). Manual: Estrategias de evaluación pericial en abuso sexual infantil. Santiago: Servicio Nacional de Menores, Gobierno de Chile.

Ministerio de Salud. (1998). Guía para la detección y respuestas al maltrato físico y abuso sexual en niños y adolescentes en los servicios de urgencia. Santiago, Chile: Ministerio de Salud.

Miranda, J. \& Sanza, V. (2004). Estudio descriptivo comparativo del test HTP en niños abusados sexualmente y niños sin sospecha de abuso sexual. Manuscrito no publicado, Santiago, Chile

Olea. C. \& Rivera J. (2007). Peritaje en víctimas de abuso sexual infantil: un acercamiento a la práctica chilena. Cuadernos de Neuropsicología, 1(3). Recuperado el 26 de mayo del año 2008 de: http:// pepsic.bvs-psi.org.br/scielo.php?script=sci_arttext\&pid=S0718$41232007000300011 \& \operatorname{lng}=\mathrm{pt} \& \mathrm{nrm}$

Policía de Investigaciones de Chile. (2003). Centro de Asistencia a víctimas de atentados sexuales, CAVAS Metropolitano. 16 años de Experiencia. Santiago: Versión/Producciones Gráficas Ltda.

Servicio Nacional de Menores. (2008). Género, Maltrato e Infancia: Manual dirigido a los Equipos de proyectos especializados en maltrato y abuso sexual grave de la red de SENAME. Recuperado el 21 de junio del año 2008 de http://www.sename.cl/wsename/otros/proteccion/Manual_generomaltrato_infancia.pdf

Fecha de recepción: 03 de mayo de 2010.

Fecha de aceptación: 06 de julio de 2010. 